\title{
Análise das Principais Espécies de Scolytidae Coletadas em Floresta Primária no Estado do Amazonas
}

\author{
Raimunda L. S. Abreu ${ }^{1}$, Cláudio R. V. Fonseca² e Eli N. Marques ${ }^{3}$ \\ ${ }^{1}$ Coordenação de Pesquisas em Produtos Florestais, INPA, Av. André Araújo, \\ 1756, 69011-970, Manaus, AM. \\ ${ }^{2}$ Coordenação de Pesquisas em Entomologia, INPA, Av. André Araújo, 1756, \\ 69011-970, Manaus, AM. \\ ${ }^{3}$ Departamento de Silvicultura e Manejo, UFPR, Rua Bom Jesus 650, \\ Juvevê, 80035-010, Curitiba, PR.
}

\author{
An. Soc. Entomol. Brasil 26(3): 527-535 (1997) \\ Analysis Species of Scolytidae Collected in the Primary Forest \\ of the State of Amazonas
}

\begin{abstract}
A survey on the occurrence of insecta of the Scolytidae family (Coleoptera) was carried out in the area of primary forest of the forest Reserve Adolpho Ducke, with flight traps Escolitídeo/Curitiba and Marques/Carrano/ Abreu, installed at height of $1 \mathrm{~m} ; 3 \mathrm{~m} ; 5 \mathrm{~m} ; 7.5 \mathrm{~m}$, and $10 \mathrm{~m}$. The collected data were evaluated quantitatively and qualitatively. From the 7974 Scolytidae insects collected, 4131 (51.8\%) were captured in the Escolitídeo/Curitiba traps and $3843(48.2 \%)$ in the Marques/Carrano traps. The genus Xyleborus was responsible for more than $50 \%$ of the total collection and the principal species were: Xyleborus affinis Eichhoff was predominant during the whole period of the experiment, Xyleborus $\mathrm{sp}_{1}$, Premnobius cavipennis Eichhoff, Cryptocarenus heveae (Hagedorn), Cryptocarenus diadematus Eggers, Monarthrum $\mathrm{sp}_{1}$, Hypothenemus eruditus Westwood, Hypothenemus obscurus Fabricius, and Xylosandrus compactus Eichhoff.
\end{abstract}

KEY WORDS: Insecta, Coleoptera, survey, primary forest, ethanol trap.

RESUMO - Em área de floresta primária da Reserva Florestal Adolpho Ducke, foi feito o levantamento da ocorrência de insetos da família Scolytidae (Coleoptera), utilizando-se as armadilhas Escolitídeo/Curitiba e Marques/ Carrano/Abreu, instaladas em alturas de $1 \mathrm{~m} ; 3 \mathrm{~m} ; 5 \mathrm{~m} ; 7,5 \mathrm{~m}$ e $10 \mathrm{~m}$. Os dados de coleta foram avaliados quantitativa e qualitativamente. Dos 7.974 escolitídeos coletados, 4.131 (51,8\%) o foram com a armadilha Escolitídeo/Curitíba e 3.843 $(48,2 \%)$ com a Marques/Carrano. Os dados revelam a existência de 14 gêneros e 168 espécies. O gênero Xyleborus foi responsável por mais de 50\% das coletas e as principais espécies capturadas foram: Xyleborus affinis Eichhoff, que predominou durante o período do experimento, Xyleborus $\mathrm{sp}_{1}$, Premnobius cavipennis Eichhoff, Cryptocarenus heveae (Hagedorn), Cryptocarenus diadematus Eggers, Monarthrum $\mathrm{sp}_{1}$, Hypothenemus eruditus Westwood, Hypothenemus obscurus Fabricius e Xylosandrus compactus Eichhoff.

PALAVRAS-CHAVE: Insecta, Coleoptera, levantamento, mata nativa, arma dilha de etanol. 
Os representantes da família Scolytidae são considerados brocas de tecidos de angiospermas e coníferas. Eles têm se diversificado, diferindo quanto às plantas hospedeiras, com relação ao tecido envolvido e ao tempo gasto com uma dada espécie florestal (Atkinson 1985).

O ciclo biológico da maioria das espécies de Scolytidae transcorre dentro da árvore. Adultos sexualmente maduros invadem os tecidos susceptíveis da planta, constroem galerias e aí acasalam. A oviposição ocorre nas galerias e o desenvolvimento larval e pupal, em geral, sucede-se na mesma galeria ou nos tecidos adjacentes a esta. Usualmente, após a emergência, os adultos voam em busca de um novo hospedeiro para iniciar um novo ciclo (Atkinson 1985).

De acordo com seus hábitos alimentares, os escolitídeos foram divididos por Furniss \& Carolin (1977) em dois grupos distintos: besouros de casca (bark beetles) e besouros ambrósia (ambrosia beetles).

Os besouros de casca são considerados insetos primários porque causam a morte da planta hospedeira, uma vez que constroem galerias entre a casca e o lenho das árvores, e alimentam-se do tecido vascular. As citações sobre este grupo no Brasil foram feitas, entre outros, por Bleicher \& Bleicher (1977), Carrano-Moreira (1985) e Marques (1984, 1989).

Os besouros ambrósia recebem esta denominação porque alimentam-se de fungos por eles transportados e cultivados na madeira. Constroem galerias profundas, atingindo o alburno e muitas vezes o cerne. Segundo Beaver (1976), todas as espécies de besouros ambrósia pertencem à tribo Xileborini, e pelo fato destas espécies selecionarem para seu ataque hospedeiros enfraquecidos, decadentes, árvores recémabatidas, madeira verde e madeira úmida, além de restos de exploração madeireira, são considerados insetos secundários, uma vez que não causam a morte da planta (Browne 1962, Saunders \& Knoke 1967, Dyer 1963, Beaver 1976).

Estudos sobre biologia, ecologia e comportamento alimentar desses insetos têm sido amplamente realizados (Wood 1982). Todavia, para a escolitidofauna do Brasil e da região amazônica, existem lacunas quanto a estes aspectos. Por este motivo, foi realizado um levantamento das espécies da família Scolytidae presentes em área de floresta primária com o propósito de fornecer subsídios para que futuras pesquisas adicionem maiores informações sobre estes insetos, que são importantes do ponto de vista econômico.

\section{Material e Métodos}

Este trabalho foi realizado na Reserva Florestal Adolfo Ducke, localizada no Km 22 da rodovia AM 010 (0308' S e 59 52'40”'W), compreendendo uma área de 10.072ha, cujos dados de vegetação foram descritos por vários autores, entre eles Lechthaler (1956), Aubréville (1961) e Magalhães \& Alencar (1979); o clima é do tipo Afi, de acordo com a classificação de Koppen (Koppen 1948). Na amostragem dos insetos, foram utilizadas as armadilhas de impacto Escolitídeo/Curitiba modificada (Carrano-Moreira 1985) e Marques/Carrano/Abreu. Esta armadilha é resultante da modificação efetuada no painel de impacto do modelo Marques/Carrano (Carrano-Moreira \& Pedrosa-Macedo 1994), originalmente em plástico, que foi substituído por folha de alumínio.

As armadilhas foram instaladas em alturas de $1 \mathrm{~m}, 3 \mathrm{~m}, 5 \mathrm{~m}, 7,5 \mathrm{~m}$ e $10 \mathrm{~m}$, com cinco repetições por altura, perfazendo um total de 25 Escolitídeo/Curitiba modificada e 25 Marque/Carrano/Abreu. Elas ficaram dispostas em dez linhas alternadas, ou seja, uma linha com cinco armadilhas de um modelo, em diferentes alturas, e a outra linha igualmente com o outro modelo na mesma disposição. Tanto a distância entre as linhas como entre colunas foi de 30 metros.O atrativo usado foi o álcool comercial. Para evitar predação e conservar os insetos, foi colocado álcool 30\% com detergente dentro do frasco coletor, renovado semanalmente, quando também era feita a renovação do 
atrativo.

As coletas foram realizadas com freqüência semanal, durante oito meses, com início em março e término em outubro de 1993, compreendendo uma parte da estação chuvosa, o período de transição e uma parte da estação seca. Para análise, os dados foram agrupados mensalmente. A identificação dos escolitídeos foi feita utilizando-se o trabalho de Wood (1992) e por comparação direta com exemplares já anteriormente identificados na Universidade Federal do Paraná e depositados na Coleção Sistemática de Invertebrados do Instituto Nacional de Pesquisas da Amazônia. A análise quantitativa foi feita através da contagem direta dos exemplares e a frequiência de coleta foi obtida calculando-se as percentagens de indivíduos de cada espécie em relação ao número total de indivíduos capturados (Silveira Neto et al. 1976).

\section{Resultados e Discussão}

Foram capturados 7.974 exemplares de Scolytidae, sendo 4.131 com a armadilha Escolitídeo/Curitiba, representando 51,8\%, e $3.843 \mathrm{com}$ a Marques/Carrano/Abreu, representando $48,2 \%$. Os dados revelam a existência de 14 gêneros e 168 espécies (Tabela $1)$.

Do total de insetos capturados com a armadilha Marques/Carrano/Abreu, destacam-se pela freqüência as espécies Xyleborus affinis Eichhoff, representando 52,4\% (2014 ind.); Premnobius cavipennis Eichhoff, 8,2\% (314 ind.); Cryptocarenus heveae (Hagedorn), 7,8\% (300 ind.); Xyleborus $\mathrm{sp}_{1}$ Eichhoff, 3,5\% (136 ind.); Xyleborus spp., $3.0 \%$ (116 ind.); Monarthrum $\mathrm{sp}_{1}$ Kirsch, 2,6\% (100 ind.); Hypothenemus eruditus Westwood, 2,5\% (99 ind.); Xylosandrus compactus Eichhoff, 2,5\% (98 ind.); Hypothenemus obscurus Fabricius, 2,5\% (98 ind.); Monarthrum spp., 2,0\% (79 ind.); Sampsonius spp. Eggers, 1,8\% (69 ind.); e Amphicranus spp. Erichson, 1,2\% (47 ind.). As demais espécies representaram 9,7\% (373 ind.). Na armadilha Escolitídeo/Curitiba, $X$. affinis representou 55,2\% (2.279 ind.); $C$. heveae, 9,1\% (376 ind.); P. cavipennis, 6,6\% (272 ind.); Xyleborus $\mathrm{sp}_{1}, 3,7 \%$ (154 ind.); Xyleborus spp., 3,4\% (139 ind.); $X$. compactus, 2,6\% (106 ind.); H. obscurus, 2,4\% (99 ind.); Monarthrum spp., 2,2\% (90 ind.). H. eruditus, $1,9 \%$ (76 ind.); Sampsonius spp., $1,8 \%$ (74 ind.); Monarthrum $\mathrm{sp}_{1}, 1,5 \%$ (61 ind.); e $C$. diadematus Eggers, 1,4\% (57 ind.). As demais representaram 8,4\% (349 ind.) (Tabela 1).

$\mathrm{Na}$ armadilha Marques/Carrano/Abreu a $1 \mathrm{~m}$ do solo, as espécies que se destacaram foram: X. affinis com 896 indivíduos; Xyleborus $\mathrm{sp}_{1}$, com 52 indivíduos; Monarthrum $\mathrm{sp}_{1}$, com 51 indivíduos; $P$. cavipennis, com 41 indivíduos; e $X$. compactus, com 30 indivíduos. A $3 \mathrm{~m}$, as espécies destacadas foram: X.affinis, com 609 indivíduos; P. cavipennis, com 65; Xyleborus $\mathrm{sp}_{1}$, com 56. A $5 \mathrm{~m}$ e $7.5 \mathrm{~m}, X$. affinis, com 248 e 167, respectivamente; $C$. heveae, com 75 e 131, respectivamente; $P$. cavipennis, com 67 e 66, respectivamente. A 10 m, X.affinis, com 94; P. cavipennis, com 75; e $C$. heveae, com 63 . Na armadilha Escolitídeo/Curitiba ainda houve a superioridade numérica da espécie $X$. affinis, com 1311 indivíduos a 1m, 561 a 3m, 181 a $5 \mathrm{~m}$ e 120 a $7,5 \mathrm{~m}$, diminuindo consideravelmente a 10 metros do solo (106 ind.), quando houve o aumento de $C$. heveae, com 149 indivíduos. O número de indivíduos da espécie $P$. cavipennis aqui foi diretamente proporcional à altura (Tabela 1).

Os registros de coleta demonstram que não houve diferenciação qualitativa entre as armadilhas Escolitídeo/Curitiba e Marques/ Carrano/Abreu, no entanto, foi observada uma pequena superioridade numérica a favor da primeira armadilha (Tabela 1; Fig. 1).

A maioria das espécies capturadas pertence às tribos Xyleborini (Xyleborus, Premnobius e Sampsonius) e Corthylini (Amphicranus, Corthylus, Microcorthylus, Tricolus e Monarthrum) predominantes em regiões tropicais, com hábitos xilomicetófagos, ou seja, alimentam-se de fungos por eles cultivados dentro da planta. Outra tribo encontrada foi Cryphalini, com os gêneros 


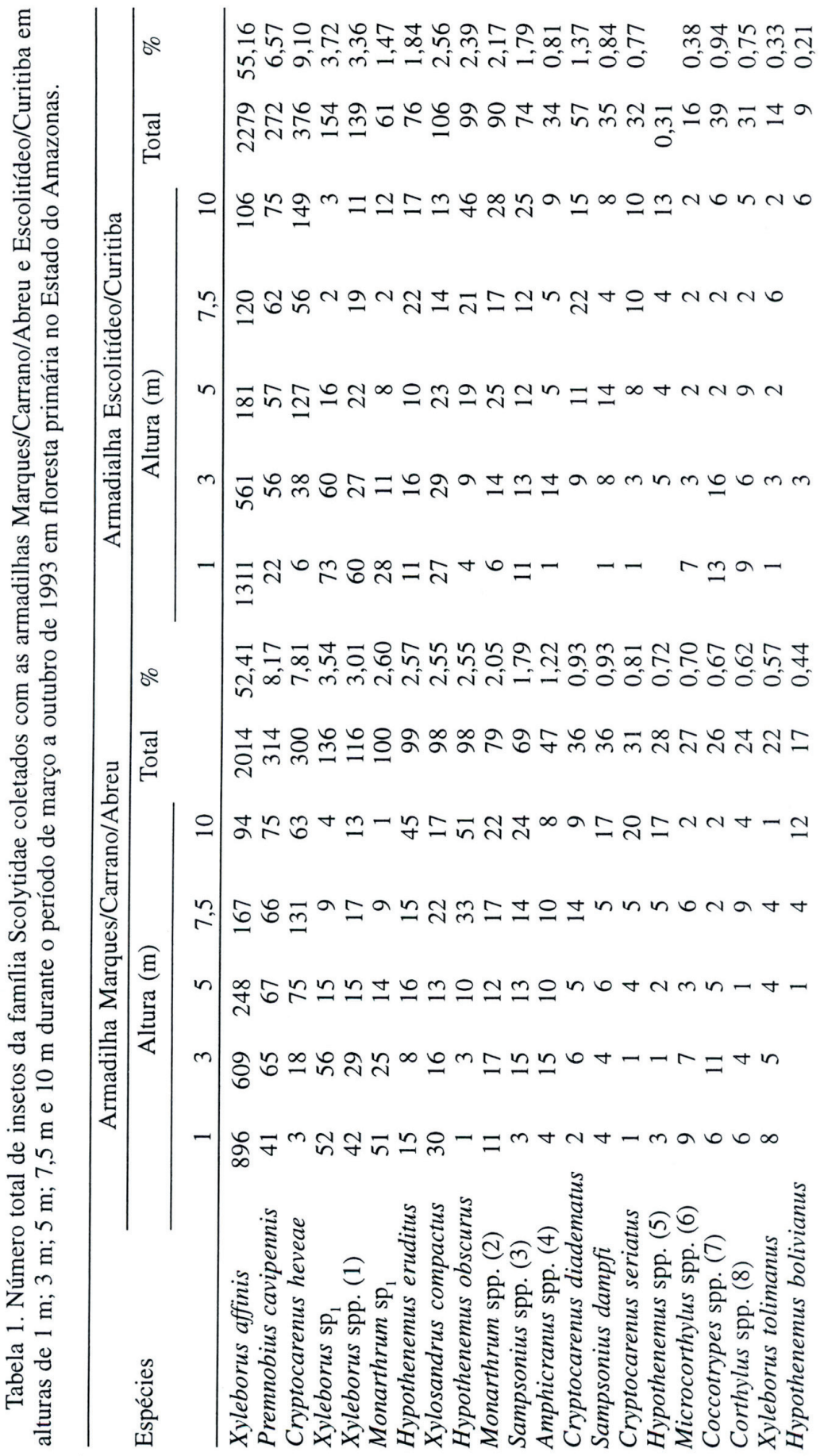




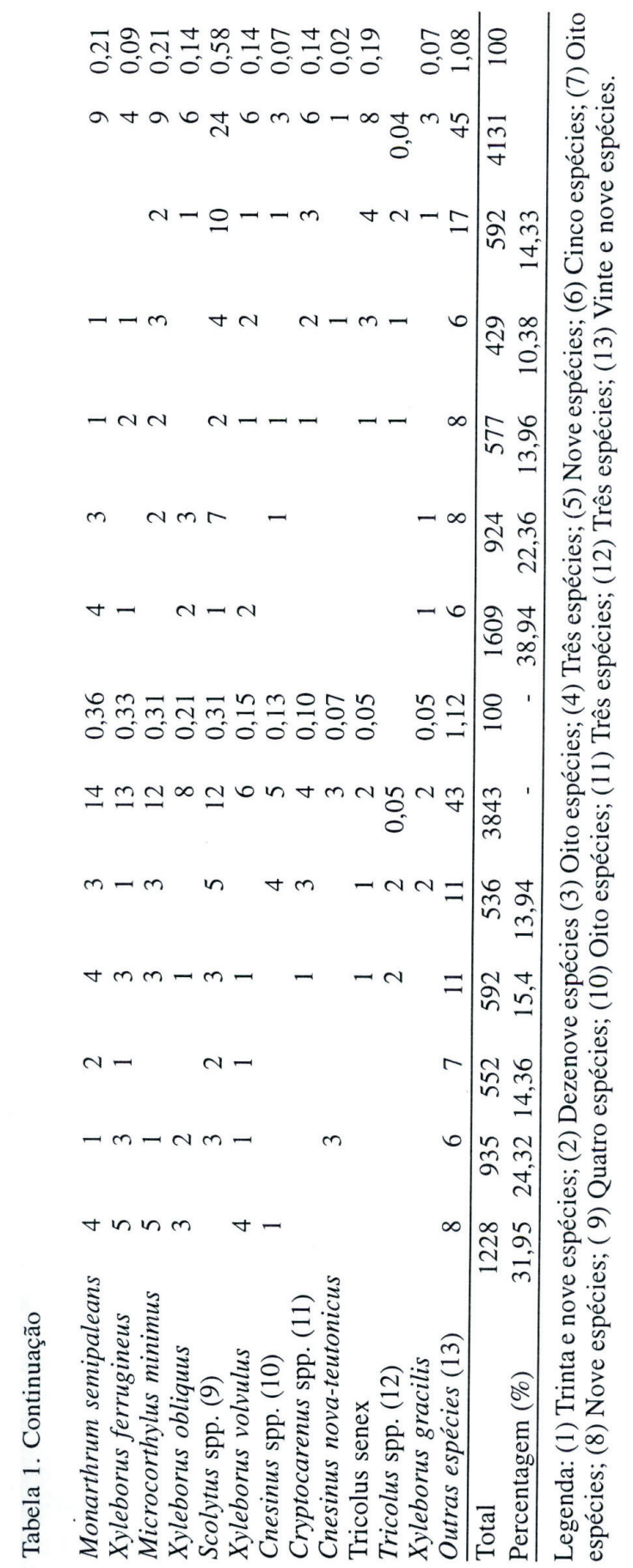


Cryptocarenus e Hypothenemus, também comuns em regiões tropicais, com hábitos alimentares bastante variados, alimentandose de medulas e brotos (mielófagos), floemas (floéfagos) e xilema (xilófagos) (Wood 1982, Atkinson 1985).

Apesar do gênero Xyleborus ter apresentado o maior número de espécies nas duas armadilhas (mais de 50\%), somente $X$. affinis destacou-se, estando presente em todas as alturas, porém diminuindo conforme a armadilha se distanciava do solo (Tabela 1). Zelaya (1985) e Fletchmann (1988) também registraram a presença desta espécie em plantios de pinheiros tropicais no Município de Agudos, Estado de São Paulo, capturando maior número a $4 \mathrm{~m}$ do solo. Esta espécie influenciou sobremaneira a coleta a $1 \mathrm{~m}$ e $3 \mathrm{~m}$ do solo, tanto para a armadilha Escolitídeo/ Curitiba como para Marques/Carrano/Abreu. Se a referida espécie for excluída, percebe-se que a coleta é mais ou menos constante em termos quantitativos em todas as alturas, com um ligeiro aumento para as alturas de $5 \mathrm{~m}$ e $10 \mathrm{~m}$ para a primeira armadilha e $7,5 \mathrm{~m}$ e 10 $\mathrm{m}$ para a segunda. Apesar da influência da altura, pode-se dizer que $X$. affinis predomina na região das coletas, uma vez que há uma

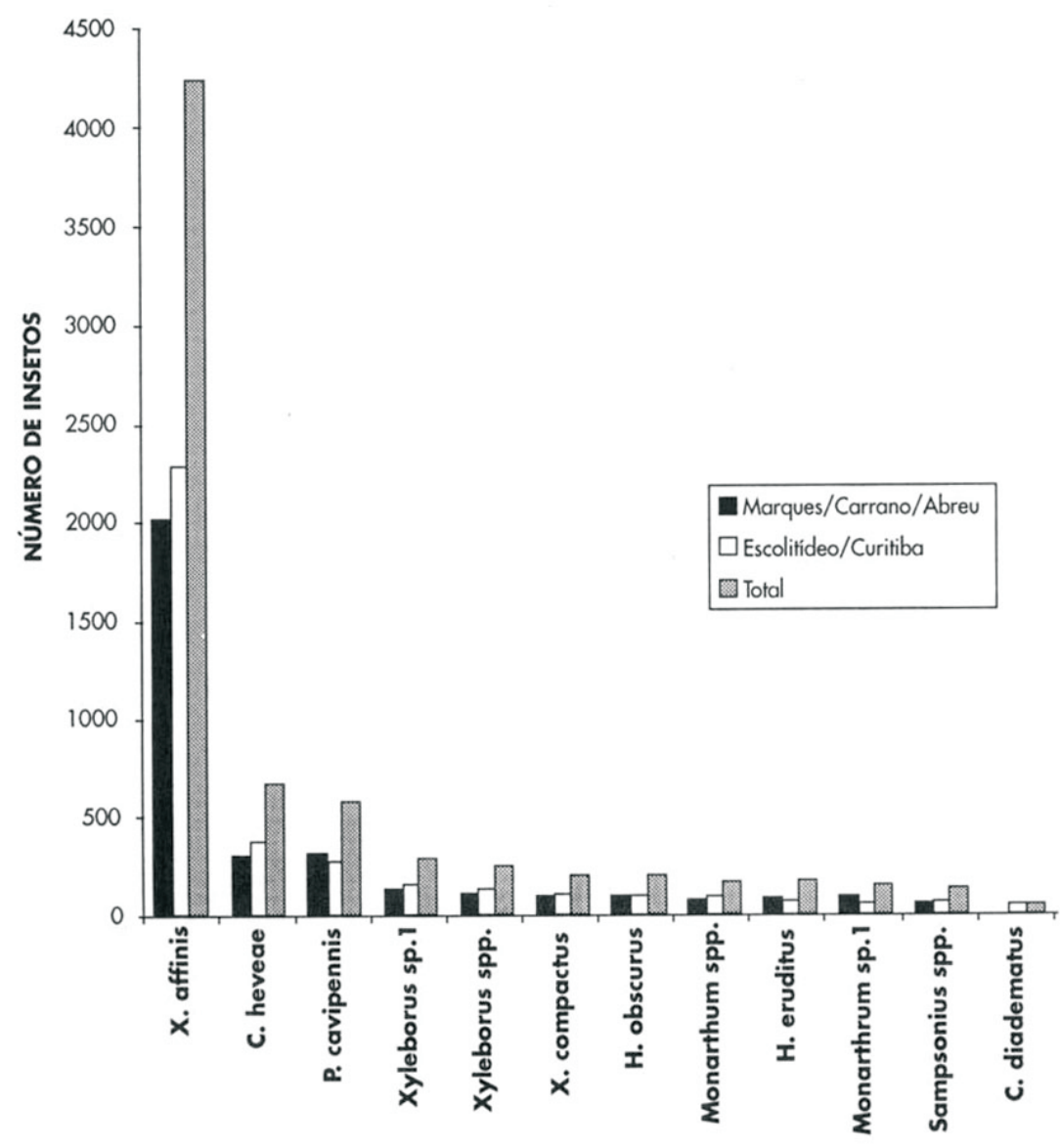

Figura 1. Principais espécies de insetos da família Scolytidae (Insecta: Coleoptera) coletadas com as armadilhas Marques/Carrano/Abreu e Escolitídeo/Curitiba em Floresta Primária no Estado do Amazonas. 
diferença muito grande entre ela e as demais, inclusive quando comparadas com aquelas que apresentam os mesmos hábitos alimentares. Em florestas tropicais, sem estações definidas é normal serem encontradas muitas espécies com abundância relativa baixa (Odum 1913), porém, os resultados indicam que a família Scolytidae foge ao padrão, pois houve forte dominância de $X$. affinis. Isto pode ser justificado ou pela competição interespecífica, considerando que espécies com rápido desenvolvimento alimentam-se mais rapidamente, resultando assim na diminuição dos recursos para as outras (Rudinsky 1962), ou pelo forte tropismo desta espécie quando em presença de álcool. Portanto, sugere-se que sejam feitos novos levantamentos, utilizando-se outros tipos de armadilhas para verificar a veracidade das hipóteses. A esta espécie são atribuídos vários tipos de danos em regiões tropicais (Wood 1982). Trabalhos realizados no Brasil, prin- cipalmente no Sul (Marques 1984, 1989, Carrano-Moreira 1985), demonstram que $X$. affinis é pouco freqüente naquela região, porém Beaver (1976) em estudo realizados em floresta nativa no Estado do Mato Grosso e Flechtmann (1988), no Estado de São Paulo, ressaltam a sua importância nessas áreas, demonstrando que quanto mais quente e úmida a região, melhor sua adaptação.

A espécie $X$. ferrugineus, considerada como das mais importantes e abundantes em regiões tropicais, inclusive sendo vetor do fungo Ceratocyistis fimbriata (Ellis \& Halsted), causador da morte de várias plantas (Browne 1962, Beaver 1976, Wood 1982), teve baixa frequiência (Tabela 1), corroborando o trabalho realizado por Abreu (1992) em madeiras da região Amazônica, onde sua incidência também foi menor.

C. heveae foi a segunda espécie mais capturada, principalmente com a armadilha Escolitídeo/Curitiba, destacando-se também em todas as alturas, porém com uma peculiaridade: sua abundância é diretamente proporcional à distância do solo, ou seja, a partir de 5 metros (Tabela 1), concordando com trabalhos realizados por Flechtmann
(1988). Sua ocorrência em regiões tropicais também é grande e de relevante importância, pois ela costuma atacar a região da medula dos galhos de árvores (Wood 1982) e sementes (Browne 1963). Beaver (1976) encontrou esta espécie brocando galhos de várias essências florestais no Estado do Mato Grosso.

A terceira espécie mais coletada foi $P$. cavipennis, encontrada em todas as alturas, mas a armadilha Marques/Carrano foi mais eficiente na sua captura (Tabela 1). Esta espécie também possui hábitos xilomicetófagos (Wood 1982).

Do gênero Hypothenemus, destacam-se as espécies $H$. eruditus e $H$. obscurus. O que se percebe com relação a estas espécies é que a primeira foi mais capturada com a armadilha Marques/Carrano/Abreu do que com a Escolitídeo/Curitiba, e para a segunda não houve diferença (Tabela 1).

Outra espécie importante economicamente é $X$. compactus, que, apesar de ser xilomicetófaga, costuma desenvolver-se em galhos de árvores sadias e vigorosas (Wood 1982); inclusive foi detectada causando o secamento da copa e/ou tronco de coqueiros jovens no Pará (Mendes 1978). Foi encontrada em todas as alturas, sendo mais capturada com a armadilha Escolitídeo/Curitiba que com a Marques/Carrano/Abreu (Tabela 1).

Um ponto importante observado é que o número de insetos capturados com estas armadilhas, pequeno em relação aos capturados com armadilhas similares em reflorestamento noutras regiões do Brasil (Marques 1984, 1989, Carrano-Moreira 1985, Flechtman 1988), pode ser devido a grande biomassa existente na região em estudo, o que leva a se supor que haja grande concentração de etanol natural no ambiente, fator que provavelmente dilui a atratividade das armadilhas.

\section{Literatura Citada}

Abreu, R. L. S. de 1992. Estudo da ocorrência de Scolytidae e Platypodidae em madeiras da Amazônia. Acta 
Amazonica 22: 413-420.

Atkinson, T. H. 1985. Los generos de la família Scolytidae (Coleoptera en Mexico. Resumen de su taxonomía y biología. Memoria de Los Simposia Nacionales de Parasitologia Florestal II y III. Pub. Esp. ${ }^{\circ}$ 46. Sec. de Agric. y Rec. Hidr., México, D. F.

Aubréville, A. 1961. Étude ecologique des principales formations vegetales du Brésil et contribuition a la connaissance des forests de L'Amazonie Bresiliene. Cent. Tech. Forest. Trop., France, 268p.

Beaver, R. A. 1976. Biological studies of Brazilian Scolytidae and Platypodidae (Coleoptera). V. The tribe Xyleborini. Z. Ang. Ent. 80: 15-30.

Bleicher, J. \& E. Bleicher. 1977. Identificação de coleópteros (Scolytidae) que atacam a ameixeira e a macieira em Santa Catarina. Indicação de Pesquisa, EMPASC, Florianópolis, n 10.

Browne, F. G. 1962. Notes on Xyleborus ferrugineus (F). In: 5th Rep. W. Afr. Timb. Borer Res. Unit (1961-1962): 4755.

Browne, F. G. 1963. Notes on the habits and distribution of some ghanaian bark beetles and ambrosia beetles (Coleoptera: Scolytidae and Platypodidae). W. Afr. Timb. Borer Res. Unit. (1961-1962): 229226.

Carrano-Moreira, A. F. 1985. Análise faunística de Scolytidae em comunidade florestais no Estado do Paraná. . Tese de mestrado, UFRPE, Recife, 90p.

\section{Carrano-Moreira, A. F. \& J. H. Pedrosa-} Macedo. 1994. Levantamento e análise faunística da família Scolytidae (Coleoptera) em comunidades florestais no Estado do Paraná. An. Soc. Entomol.
Brasil 23:115-126.

Dyer, E. D. A. 1963. Attack and brood production of Ambrosia beetles in logging debris. Can. Entomol. 95: 624631.

Flechtmann, C. A. H. 1988. Altura de vôo de Scolytidae em reflorestamento com pinheiros tropicais na região de Agudos, Estado de São Paulo. Tese de mestrado, USP/ESALQ, Piracicaba, 132p.

Furniss, R. L. \& V. M. Carolin. 1977. Western forest insects. USDA, Misc. Publ., n 1339, 654p.

Köppen, W. 1948. Climatologia: con un estudio de los climas de la terra. México. Fondo Cult. Econ. 479p.

Lechthaler, R. 1956. Inventário das árvores de um hectare de terra firme da zona "Reserva Florestal Ducke", Município de Manaus. INPA, Sér. Botânica 3: 1-7.

Magalhães, L. M. \& J. C. Alencar. 1979. Fenologia do pau-rosa (Aniba duckei Kostermans) Lauraceae, em floresta primária da Amazônia Central. Acta Amazônica 9: 227-237.

Marques, E. N. 1984. Scolytidae e Platypodidae em Pinus taeda. Tese de mestrado, UFPR, Curitiba, 65p.

Marques, E. N. 1989. Índices faunísticos e grau de infestação por Scolytidae em madeira de Pinus spp. Tese de doutorado, UFPR, Curitiba, 103p.

Mendes, A. C. de B. 1978. Insetos associados ao cacaueiro na Amazônia (reconhecimento e controle). Comissão Executiva do Plano da Lavoura Cacaueira. Dep. Esp. Amazônia, p. 24.

Odum, E. P. 1913. Ecologia. Tradução Christopher J. Tribe; supervisão de 
tradução Ricardo Iglesias Rios. Rio de Janeiro: Discos CBS 1985. pp 233-281.

Rudinsky, J.A. 1962. Ecology of Scolytidae. Annu. Rev. Entomol. 7: 327-344.

Saunders, J. L. \& J. K. Knoke. 1967. Diurnal emergence of Xyleborus ferrugineus (Coleoptera: Scolytidae) from cacao trunks in Ecuador and Costa Rica. Ann. Entomol. Soc. Am. 60: 10941096.

\section{Silveira Neto, S., O. Nakano, D. Barbin \&} N. A. Villa Nova. 1976. Manual de ecologia dos insetos. São Paulo, Ed.
Agronômica Ceres Ltda., 419p.

Wood, S. L. 1982. The bark and Ambrosia beetles of North and Central America (Coleoptera: Scolytidae), a taxonomic monograph. G. B. Nat. Mem., 6:1-1360.

Zelaya, M. R. M. 1985. Observações sobre o comportamento de Xyleborus spp. (Coleoptera: Scolytidae) em florestas de Pinnus spp. na região de Agudos, Estado de São Paulo.Tese de mestrado, USP/ ESALQ, Piracicaba, 88p.

Recebido em 16/05/97. Aceito em 20/10/97. 